\title{
Realization of Fractional Order Elements
}

\author{
Avishek Adhikary ${ }^{1} \cdot$ Munmun Khanra $^{2} \cdot$ Jayanta Pal $^{3} \cdot$ Karabi Biswas $^{4}$
}

Received: 24 May 2016/ Accepted: 11 April 2017/Published online: 20 April 2017

(C) Indian National Academy of Engineering 2017

\begin{abstract}
Fractional order (FO) element (FOE) is the building block for realization of FO systems, an important research domain. However, the realization of FO system is still a challenge. Till date, no FOE or fractor is available in the market. However, many researchers have developed various types of FOE, multi-component and single component, over past 50 years or more. This paper reviews some significant research work, along with their success and limitations, in the field of FOE realization. Also, it discusses the chronological development and a brief comparative study to present an overall idea on current stage of FOE research to the readers.
\end{abstract}

Keywords Constant phase - Fractional order circuits . Fractional order systems $\cdot$ Fractor realization

Avishek Adhikary

avishek.adhikary@iitkgp.ac.in

Munmun Khanra

munmunkhanra@gmail.com

Jayanta Pal

jpal@iitbbs.ac.in

Karabi Biswas

karabi@ee.iitkgp.ernetin

Department of EE, IIT Kharagpur, Kharagpur, India

2 Department of EIE, NIT Silchar, Silchar, India

3 Department of EE, IIT Bhubaneswar, Bhubaneswar, India

4 Department of EE, IIT Kharagpur, Kharagpur, India

\section{Introduction}

Fractional order element (FOE) is the electrical element whose impedance function is described by fractional order (FO) calculus. The concept of FO calculus is as old as integer order (IO) calculus (Oldham and Spanier 1974) but, remained less practiced for centuries. However by 1980, literature started to claim that FO calculus is more apt to catch complex system dynamics as it has more flexibility in differential order. The next few decades witness an explosion in the application of FO calculus in system modelling (Machado and Jesus 2004). From any distributive natural processes like diffusion (Sierociuk et al. 2015), conduction (Žecov and Terpák 2015), volcanology (Fortuna et al. 2014), earthquake (Lopes and Machado 2016) to material characterization including solid composite (Macdonald 1987), viscoelastic (Arikoglu 2014); from modeling of virus (Pinto and Carvalho 2014) and human organs (Ionescu et al. 2011) to different electrochemical cells (Freeborn et al. 2015) and sensors (Adhikary et al. 2016); from biochemistry and biomedicine (Freeborn 2013) to bioengineering (Magin 2004) etc. The FO modelling became an emerging tool in science and engineering (Caponetto et al. 2010; Ortigueira 2011). This led to wide spread research interest in FO control (Podlubny 1999; Monje et al. 2010; Das 2010; Caponetto et al. 2010; Baleanu et al. 2012; Bandyopadhyay and Kamal 2014) and FO signal processing (Magin et al. 2011). The arbitrariness in the order of FO systems offers additional tuning parameters in the FO controller making it a potential candidate for controlling complex systems like diffusion (Ozdemir and Iskender 2010); pressurized heavy water reactor (Saha et al. 2010); wind energy (Melicio et al. 2010); twin rotor MIMO system (Mishra and Purwar 2014); mechatronics, biological systems, and many more 
(Monje et al. 2010). Besides, a number of FO electronic systems, like FO oscillators (Radwan et al. 2008), FO filters (Adhikary et al. 2016; Tripathy et al. 2013, 2015; Tseng 2007; Soltan et al. 2015; Freeborn et al. 2015), FO PLL (Tripathy et al. 2015), and many others (Biswas et al. 2006; Mondal and Biswas 2011; Adhikary et al. 2016) have been developed and have shown promising results.

But, this rapid development in theoretical area yet not reflected in practice for the unavailability of commercial FO element (FOE). However, last few decades has witnessed many successful realization of FOE. All such realizations are broadly classified into two categories: (a) Single component and (b) Multi-component realization. Each of them has some merits and some limitations, and neither of them is standardized yet. Here, we discuss these realizations in detail and compare their features and limitations. The "Fractional Order Element" discusses how FOE is defined in various literature. In the "Realization of FOE", first, various multi-component fractors and then, various single component fractors are described. The final section is "Conclusion".

\section{Fractional Order Element}

Fractional order element is the electrical element whose impedance function follows FO differential equation. In $s$ plane it is represented as,

$Z_{F}(s)=\frac{R}{(\tau s)^{\alpha}} \equiv \frac{1}{C_{F} s^{\alpha}} \equiv \frac{Q}{s^{\alpha}} \equiv \frac{1}{F s^{\alpha}}$

The above expressions are most common representations for FOE, available in literature. Concept of FOE was first introduced by Cole brothers in (1941) for modelling of dielectrics, and was represented like $Z_{F}(s)=R /(\tau s)^{\alpha}$. Here, $R$ is a constant of unit $\Omega, \tau$ is another constant of unit second and $\alpha$ is fractional number. Later, such representation technique is followed in various literature and software algorithm (Macdonald 2015).

But, many researchers prefer $\mathrm{FOE}$ as 'fractional capacitor' and presents it as, $Z_{F}(s)=1 / C_{F} s^{\alpha}$. Here also, $\alpha$ is a fractional number, but $C_{F}$ is termed as fractional capacitance and its unit is $F / s^{1-\alpha}$ (Westerlund and Ekstam 1994). The main advantage is of such realization is its resemblance to the conventional capacitor. However, the unit of coefficient is now of fractional order which is not easy to interpret.

Now, by the term fractional capacitor, they are actually envisioned as an element between capacitor and resistor. However, FOE with + ve phase is also not uncommon (viz. "Realization of FOE"). So researchers now adopt more generalized terms like FOE or fractance device and presents $Z_{F}$ as $Q / s^{\alpha}$, where, $Q$ is named as characteristic constant (Mondal and Biswas 2013) and its unit is $\Omega / s^{\alpha}$.

In some works (Bohannan 2000; Adhikary 2015; Adhikary et al. 2015), FOE has been termed as 'fractor'. Sometimes fractor is presented as, $Z_{F}=1 / F s^{\alpha}$. Here, the coefficient $F$ is termed as fractance and its unit is $V s^{\alpha}$. Main advantage of this terminology is that it is in sync with resistor, capacitor and inductor.

\section{FOE as Constant Phase Element (CPE)}

In frequency domain, the FOE can be presented as,

$Z_{F}(j \omega)=\frac{1}{F(j \omega)^{\alpha}}=\frac{1}{F \omega^{\alpha}} \angle-\frac{\alpha \pi}{2}$

So the fractor phase, $\phi_{F}=-\alpha \pi / 2$, is independent of frequency and ideally remains constant at $-\alpha \pi / 2$ value at any frequency. Hence, FOE or fractor is often called as constant phase element (CPE) (Biswas et al. 2006). Actually, resistor, capacitor, inductor and FOE- all are CPE (as $\alpha=0$, FOE becomes resistor, $\alpha=1$, FOE becomes capacitor, $\alpha=-1$, it is inductor), but only fractor can have phase other than $0^{\circ}$ or $\pm 90^{\circ}$ by varying its $\alpha$.

\section{Pseudo-CPE and Constant Phase Zone}

The ideal FOE shows constant phase (CP) for any frequency, but not the practically realized FOE. For practically realized FOE, phase is constant for a limited frequency zone and such zone is called $\mathrm{CP}$ zone (CPZ). For this reason, such practically realized FOE is sometime called pseudo-FOE or pseudo-CPE (Hirschorn et al. 2010). Even within that CPZ, the phase oscillates to some extent. The measure of this oscillation is termed here as phase ripple.

\section{What is the Physical Interpretation of Fractional Power, $\alpha$, in Unit of Fractance, $F$ ?}

It is shown above, whatever is the presentation, coefficient of FOE $\left(C_{F}, Q\right.$, or $\left.F\right)$ has a variable power $\alpha$ in their unit. E.g, unit of fractance, $F$ is $\mho s^{\alpha}$. So, if $\alpha$ varies, the unit will also vary. Like, if $\alpha=0.2$, the unit is $v s^{0.2}$, if $\alpha=0.5$, the unit is $\mho s^{0.5}$. This may create a confusion that how, a particular physical variable ( $F$ here) can have unit of variable dimensions. This is explained like this: The FOE is not the name of a particular element rather a group of elements- FOE with different $\alpha$ is its different members. So, the fractance, $F$ is also not a particular physical variable, rather, a group of physical variables. Or in other words, $F$ with different $\alpha$ is different variables under same category. That is why each of them has unit of their own dimensions. 


\section{Realization of FOE}

FOE is mentioned by Cole in (1941). After that, past seventy-five years have witnessed a number FOE realizations which are broadly classified in two parts: multicomponent and single component realization. ${ }^{1}$

\section{Realization of Multi-component FOE}

Early Multicomponent FOE: In 1950, Darlington developed a method for determination of the best possible approximation and realization of a constant phase difference function, over a desired frequency band, using a pair of phase shifting networks (Darlington 1950). The phase shifters achieve the desired CP and number of all-pass sections determine the $\mathrm{CP}$ zone. However, this methods requires various inductors and capacitors; and hence, is difficult to be realized. In 1961, Douglas realized CP functions cascading R-C blocks (Douglas 1961) only. The advantage of this scheme is that each block contains only two resistors and a capacitors and much less number of blocks are required compared to Darlington's. This work describes defined design technique for achieving desired CP, CP zone, and phase ripple. In 1963, Lerner made the realization even simpler by putting only RC parallel in each blocks (Lerner 1963). Lerner's work also introduces compensation impedances to reduce phase error at the boundaries of CPZ. All these work are based on placing poles in proper positions to have constant phase immittance. Detailed analysis is given in (Lerner 1963).

RC Ladder based FOE: In 1964, Carlson et al. generalized the pole placement process by regular Newton process and showed that a fractional capacitor (capacitive FOE) is emulated in the driving point impedance of an infinite RC ladder (Carlson and Halijak 1964). This work also established the physical realizability of any FOE by truncated ladder. However, it requires arbitrary resistors and capacitors and, thus, posing difficulty in realization. Such difficulty is partially overcome by Dutta Roy et al. (1966) by offering two schemes for realizing ladder based CPE. In one scheme they used identical $\mathrm{T}$ networks (grounded $\mathrm{C}$ at limb and $\mathrm{R}$ at hands) in cascade and in other scheme, identical parallel RC networks in cascade. In both the cases, only one variety of $R$ and $C$ is needed. But, these schemes are for $\alpha=0.5$ only. For other values of $\alpha$, various $R$ and $C$ is needed (Dutta Roy 1967). A comparative study is shown between RC ladder realization, RC lattice structure and rational function approximation

\footnotetext{
1 There is a difference between FOE and FO system (FOS). FOE is typically the realization of single fractional operator where FOS may include multiple. FOS can be realized by using appropriate FOEs or directly from their transfer functions. This paper discusses about only FOE.
}

technique (Dutta Roy 1967). It is claimed in this work, that, the elliptical approximation is best for CPE realization (in terms of larger CPZ or fewer blocks), but suffers from computational and realization complexity. In 1983, Oldham et al. adopted truncated ladder structure (using parallel $\mathrm{RC}$ ) for FOE realization and developed a defined guideline to achieve any desired $\alpha$ value. The computational advantage is that, here, the $\mathrm{R}$ and $\mathrm{C}$ of consecutive blocks are in geometric progression and progression ratios define the $\alpha$ of FOE. Like Lerner's work, Oldham et al. modified the terminal blocks to reduce error at the edges of CPZ.

FOE with pre-specified Fractance: The above work focus mainly on $\mathrm{CP}$ angle, CPZ, and phase ripple, i.e., properties related to FOE exponent $\alpha$. The particular focus on achieving a desired coefficient $(F)$ has been explored by Adhikary et al. in (2015). The work adopted the Oldham's ladder structure but modified it to achieve three specifications ( $\alpha, F$ and CPZ) simultaneously. This work realized different FOEs ( $\alpha$ : 0.2 to 0.8 ) in hardware and used them to develop other FOS (Adhikary et al. 2015). Achieving a desired $F$ is possible through fractor realization via rational approximation too.

Other hardware realized ladder based fractor: Researches for flexible fractor with fewer components is still being pursued by modifying ladder networks. In (Dorčák 2007; Dorčák et al. 2013), FO systems have been realized by FOE made of RC domino ladder and tree networks. The $\mathrm{RC}$ parameters are determined by continued fraction expansion of fractional operator. A nested ladder based FOE is reported in Sierociuk et al. (2013).

Inductive and obtuse angle fractor: The above FOEs are mainly capacitive type. Lerner (1963) and Carlson et al. (1964) have discussed how inductive FOE can be made similarly by R-L networks (replacing $\mathrm{C}$ by $\mathrm{L}$ ); however, inclusion of a number of different value inductors makes hardware realization difficult. This problem can be solved by using grounded GIC (Tripathy et al. 2015; Adhikary et al. 2015). Here, a capacitive fractor of exponent $\alpha^{\prime}$ is used to make an inductive fractor of exponent $-\alpha^{\prime}$ using GIC. The work (Adhikary et al. 2015) also extended the scope of FOE exponent beyond conventional range of \pm 1 and developed some obtuse angle FOEs $(1<|\alpha|<2)$ also. That means, CPE from any of the four quadrants can now be developed.

Besides, Charef (2006) has realized inductive fractors using RC networks. In Charef (2006), the fractional operator is first approximated by a number of pole zero pairs over a desired frequency band. Partial fraction expansion of this approximant transfer function, results in an expression representing: (i) impedance of series combination of $N+1$ parallel RC networks, for $-v e \alpha$; and (ii) admittance of parallel combination of $N+1$ series RC networks, for positive $\alpha$. Khanra et al. (2013) have demonstrated the realization of fractor $K s^{\alpha}$ where $\alpha$ can be any arbitrary real 
value. The realization is based on first, rational approximation of the fractional operator and, then, the circuit realization of the pole-zero pairs of the resultant approximant using $\mathrm{R}, \mathrm{C}$ and op-amp.

There are many such work and almost all the methods for rational approximation of fractional operator can be used for circuit realization of fractor using conventional $\mathrm{R}$, $\mathrm{C}$ and/or opamps. However, the paper restricts its scope and does not discuss about the same. There are some other multi-component fractor realization techniques using neural networks (Abbisso et al. 2001), frequency shaping (Oustaloup et al. 2000) etc., but those are still at conceptual stages.

\section{Realization of Single Component FOE}

The prime motivation behind the development of singlecomponent-FOE is to have a commercially available packaged FOE of different specifications (just like commercially available resistors or capacitors). We have already seen that FOE for different $\alpha$ and $F$ have been developed in multi-component techniques. But they contain huge number of different $\mathrm{R}$ and $\mathrm{C}$, so not very helpful for IC level fabrication yet On the other hand, many natural processes itself show FO nature (viz. "Introduction"), so if such processes can be captured in a voltage-current relationship, a single component FOE can be developed.

Fractal FOE: One such system is the fractal structure. Many literature have noticed presence of FO operator while studying fractal model (Sapoval et al. 1993). In 1997, Haba et al. first developed a fractal structure which gives prominent CP zone (Haba 1997). It is a fractal tree in star structure with level 3 of iterations. Later, they studied this fractal structure for varying fractal resistances and capacitances and showed how $\mathrm{CP}$ zone (mentioned as fractal zone also) can be varied using those resistance and capacitance (Haba et al. 2005). This fractal FOE is made on Si-substrate $(7.5 \mathrm{~cm} \mathrm{dia)}$, by photo lithography. It shows $\mathrm{CP}$ in $10^{5} \mathrm{~Hz}$ to $10^{10} \mathrm{~Hz}$ zone with $\mathrm{CP}$ angle $=36^{\circ}$ (i.e., $\alpha=0.4$ ). The reported CPZs are usually 3 to 5 decades long. Main advantages: (i) Fractal FOE are light weight, Sibased, and dry fractors; (ii) possess wide CPZ and low phase ripple $\left(< \pm 2.7^{\circ}\right)$. Main limitations: (i) FO nature at high frequency zone only; (ii) no insight how any other $\alpha$ apart from 0.4 could be achieved.

Ionic gel-Cu Electrode Based Packaged FOE: This is developed by Bohannan by putting ionic gel coated $\mathrm{Cu}-$ plate ( 1 inch square) between two square $\mathrm{Cu}$ electrodes. Gel is made by lithium nitrate and tetraethyl orthosilicate and entire system is packaged by plexiglass plate (Bohannan 2000). The developed fractor is light weight and robust and it shows wide $(10 \mathrm{~Hz}$ to $1 \mathrm{MHz}) \mathrm{CPZ}$ with $\mathrm{CP}=$ $45^{\circ}$, but has phase ripple $\left( \pm 5^{\circ}\right)$ (Bohannan 2000).
Porous polymer based electrolytic fractor: This is first reported by Biswas et al. in (2006). It is made of porous PMMA coated $\mathrm{Cu}$ or $\mathrm{Pt}$ electrodes, dipped in an ionic solution. Later, such fractors are packaged by replacing the ionic solution by ionic gel (made by agar-agar powder) (Mondal and Biswas 2013). This FOE is studied for different system parameters, i.e., dipping length, coating thickness, the solution $\mathrm{pH}$ and conductivity. It is shown that $\mathrm{CP}$ zone can be moved to high frequency zone by increasing solution $\mathrm{pH}$ or conductivity, and $\mathrm{CPZ}$ can be widened by decreasing coating thickness. However, though different $\alpha$ between 0.1 and 0.85 have been achieved, no definite correlation between $\alpha$ and any of the system parameters could have been established. The packaged fractor is smaller than the fractal FOE, cheaper and simpler to fabricate. It is successfully used in developing many FO systems (Biswas et al. 2006; Mondal and Biswas 2013; Tripathy et al. 2013, 2015). This FOE shows CPZ in between $100 \mathrm{~Hz}$ to $1 \mathrm{MHz}$ and $\mathrm{CPZ}$ is maximum 2 decades long (Mondal 2012). This is because, here, the FO nature is mainly due to the transitional behaviour from low frequency diffusion domain to high frequency electrostatic domain. The main drawbacks are: low yield rate, small CPZ and low longevity (1-6 month only).

Fractal electrode based electrolytic FOE: Such FOE is introduced in 2008 by Jesus and Machado (2009). This employs the concepts of both of the above fractors. Here, electrodes are $\mathrm{Cu}$-clad PCB with a typical fractal geometry (carpet of Sierpinski) and electrolyte is $\mathrm{NaCl}$ solution. The speciality of this FOE is the introduction of a fractal material (a sand stand within the $\mathrm{NaCl}$ solution) in the electrolytic domain also. It is shown how $\alpha$ increases with the increase of effective area of fractal structure as well as solution molarity. Here, $\alpha$ from 0.2 to 0.6 have been realized. The main drawback of this FOE is that it is bulky, spillable and it actually realizes a resistor-fractor parallel circuit not FOE itself, thus limiting its application in various FO systems.

Graphene-polymer composite based FOE: In the above fractors, fractal or porous structures were introduced to bring the effect of dispersive $R C$ blocks, as pursued in different multi-component techniques (viz. "Realization of FOE"). This concept is realized in a more structured way in the graphene-polymer composite based FOE, developed by Elshurafa et al. in (2013). In this work, di-electric of conventional parallel plate capacitor is replaced by a polymer composite, percolated with reduced graphene oxide. The properly dispersed graphene sheets in the polymer gives a number of $R C$ blocks of different relaxation times, which ultimately give FO nature. This FOE shows different $\alpha$ value (0.33-0.73) for different graphene load (12-2\% respectively). The main advantages are: (i) it is small, PCB compatible, already developed as a miniature 
device and (ii) it possess an easier way to have different $\alpha$ (by simply changing graphene loading). On the other hand, (i) the fabrication procedure is complicated and costly, and (ii) $\mathrm{CP}$ zone is very small $(400 \mathrm{~Hz}-2 \mathrm{MHz}$ only).

Ionic polymer-metal composite based electrochemical fractor: Such fractor was developed by Caponetto et al. in (2013). This also has similar structure like that of Elshurafa et al. (2013), the only difference is instead of graphenepolymer composite, here the electrolyte is Pt-polymer composite. And the platinum $(\mathrm{Pt})$ absorption time (in the membrane, during fabrication) determines the $\alpha$ of the FOE. Higher is the absorption time higher is $\alpha$ value but smaller is the CP zone. The reported variation of $\alpha$ is here $0.05-0.3$, in the frequency zone $10 \mathrm{mHz}-10 \mathrm{~Hz}$. So this is suitable for low $\alpha$ value fractor and low frequency zone. However, the fabrication process is costly and CP zone is small.

CNT-polymer composite based electrochemical fractor: This wide band fractor is reported by Adhikary et al. in (2015). It has similar structure like that of Biswas et al. (2006), the difference is that the electrodes are now coated with carbon nanotube (CNT)-polymer composite instead of porous PMMA. In PMMA based FOE, the effect of porosity does not become prominent until the coating thickness is too low. So, the CPZ is small in Biswas et al. (2006). This problem is overcome by adding $1.5 \%$ CNT loading in the polymer. This CNT based FOE shows 5 decades long CPZ ranging from $20 \mathrm{~Hz}$ to $2 \mathrm{MHz}$ with $\alpha=0.35$. The main advantage is: long CPZ, low phase ripple $\left( \pm 2^{\circ}\right.$ only) and high yield rate. The main limitation is that any variation in $\alpha$ is yet not explored in this work.

At the end one can say that, the multi-component techniques have defined guidelines to achieve particular $\alpha$ or $F$ but they are burdened by the trade off between accuracy and number of components. The single component fractors are on the other hand, advantageous in cost, and compactness, but lack defined fabrication processes to meet desired specifications. Although both types are now being used to develop many successful FO applications. In Tripathy et al. (2015) a single component packaged fractor, reported in Mondal and Biswas (2013), has been used to make a FO PLL. It is found that developed FO PLL provides faster response and lower phase error at the time of switching compared to its integer-order counterpart. On the other hand, multicomponent obtuse angle fractor, presented in Adhikary et al. (2015), has been used to develop high Q factor (more than 300) tunable FO resonators (Adhikary et al. $(2016,2016)$ ) in the $\mathrm{kHz}$ range frequency. This resonators in turn makes high $\mathrm{Q}(>10)$, high attenuation $(>30 \mathrm{~dB})$ Notch filter (Adhikary et al. 2016).

\section{Conclusion}

This paper discusses different aspects of FOE or fractor and reviews recent trends and prospects of fractor realization and the challenges ahead. The paper intends to provide some ready reference to the researchers interested to explore the possibilities in the area of FO Systems realization. Due to page constraints, it has not been possible to cite all the research work carried in this field. However, the authors acknowledge all the other contributions.

Acknowledgements This research is supported by CSIR, India under the scheme of RA; Ref. 9/81(1212)/13, EMR-I, dt:28.03.2014 and SGSBI project-2014 (Code CEI), SRIC, IIT Kharagpur.

\section{References}

Abbisso S, Caponetto R, Diamante O, Fortuna L, Porto D (2001) "Noninteger order integration by using neural networks". In: IEEE Int. Symp. Circuits Syst. (ISCAS), pp 688-691

Adhikary A, Sen S, Biswas K (2016) Practical realization of tunable fractional order parallel resonator and fractional order filters. IEEE Trans Circuits Syst I 63(8):1142-1151

Adhikary A, Sen P, Sen S, Biswas K (2015) Design and performance study of dynamic fractors in any of the four quadrants. Circuits Syst Signal Process 35(6):1909-32

Adhikary A, Kumar G, Bannerje S, Sen S, Biswas K (2016) "Modelling and performance improvement of phase-angle-based conductivity sensor". In: IEEE $1^{\text {st }}$ Int. Conf. Control, Measurement and Instrumentation (CMI 2016), Kolkata, India, pp 403-407

Adhikary A, Sen S, Biswas K (2016) "Design and hardware realization of a tunable fractional-order series resonator with high quality factor". Circuits Syst Signal Process:1-20. doi:10. 1007/s00034-016-0469-2

Adhikary A, Khanra M, Sen S, Biswas K (2015) "Realization of a carbon nanotube based electrochemical fractor". In: 2015 IEEE Int. Symp. Circuits Syst. (ISCAS), Lisbon, Portugal, pp 2329-2332

Arikoglu A (2014) A new fractional derivative model for linearly viscoelastic materials and parameter identification via genetic algorithms. Rheol Acta 53(3):219-233

Baleanu D, Machado JAT, Luo ACJ (2012) Fractional dynamics and control. Springer, Berlin

Bandyopadhyay B, Kamal S (2014) Stabilization and control of fractional order systems: a sliding mode approach. Springer, Berlin

Biswas K, Sen S, Dutta PK (2006) Realization of a constant phase element and its performance study in a differentiator circuit. IEEE Trans Circuits Syst II 53(9):802-806

Bohannan G (2000) "Application of fractional calculus to polarization dynamics in solid dielectric materials". $\mathrm{PhD}$ thesis, Monatana State Univ., Bozeman, USA

Caponetto R, Dongola G, Fortuna L, Petras I (2010) Fractional order systems: modeling and control applications. World Scientific, Singapore

Caponetto R, Graziani S, Pappalardo FL, Sapuppo F (2013) Experimental characterization of ionic polymer metal composite as a novel fractional order element. Adv Math Phys 2013:1-10 
Carlson GE, Halijak CA (1964) "Approximation of fractional capacitors $(1 / s)^{1 / n}$ by a regular Newton process". IEEE Trans. Circuits Syst. CAS-11(2): 210-213

Charef A (2006) Analogue realisation of fractional-order integrator, differentiator and fractional $\mathrm{PI}^{\lambda} \mathrm{D}^{\mu}$ controller. IEE Proc Control Theory Appl 153(6):714-720

Cole KS, Cole RH (1941) Dispersion and absorption in dielectrics 1: alternating current characteristics. J Chem Phys 9(4):341-351

Darlington S (1950) "Realization of a constant phase difference". J Bell System Technical:94-104

Das S (2010) Functional fractional calculus for system identification and controls. Springer, Berlin

Dorčák L, Terpák J, Petráš I, Dorcáková F (2007) Electronic realization of the fractional-order systems. Acta Montan Slovaca 12(3):231-237

Dorčák L, Valsa J, Gonzalez E, Terpák J, Petráš I, Pivka L (2013) Analogue realization of fractional-order dynamical systems. Entropy 15(10):4199-4214

Douglas DC (1961) "A method of designing constant-phase networks". Master's thesis, Dept. Electrical Eng., Georgia Inst. Technol., Atlanta, USA

DuttaRoy SC, Shenoy BA (1966) RC realization of a constant argument impedance. J Franklin Inst 282(5):318-329

Elshurafa A, Almadhoun M, Salama K, Alshareef H (2013) Microscale electrostatic fractional capacitors using reduced graphene oxide percolated polymer composites. Appl Phys Lett 102:232901

Fortuna L, Nunari G, Cannavo F (2014) "Further evidences of selforganized criticality in volcanology". In: Proc. Int. Conf. Fractional Differentiation and its Appl., p 6

Freeborn TJ (2013) A survey of fractional-order circuit models for biology and biomedicine. IEEE J Emerg Sel Topics Circuits Syst 3(3):416-423

Freeborn TJ, Maundy B, Elwakil AS (2015) Fractional-order models of supercapacitors, batteries and fuel cells: a survey. Mater Renew Sustain Energy 4(3):1-7

Freeborn T, Maundy B, Elwakil AS (2015) "Approximated fractional order chebbyshev lowpass filters". Mathematical Problems in Eng., Hindawi Publishing Corp. 2015:7 (Article ID 832468)

Haba TC, Ablart G, Camps T (1997) The frequency response of a fractal photolithographic structure. IEEE Trans Dielectr Electr Insul 4:321-326

Haba TC, Ablart G, Camps T, Olivie F (2005) Influence of the electrical parameters on the input impedance of a fractal structure realised on silicon. Chaos, Solitons, Fractals 24(2):479-490

Hirschorn B, Orazem ME, Tribollet B, Vivier V, Frateur I, Musianid M (2010) Constant-phase-element behavior caused by resistivity distributions in films. J Electrochem Soc 157(12):C452-C457

Ionescu CM, Machado JAT, Keyser RD (2011) Modeling of the lung impedance using a fractional-order ladder network with constant phase elements. IEEE Trans Biomed Circuits Syst 5(1):83-89

Jesus IS, Machado JAT (2009) Development of fractional order capacitors based on electrolyte processes. Nonlinear Dynam 56(1):45-55

Khanra M, Pal J, Biswas K (2013) Rational approximation and analog realization of fractional order transfer function with multiple fractional powered terms. Asian J Control 15(4):723-735

Lerner R (1963) The design of a constant-angle or power-law magnitude impedance. IEEE Trans Circuit Theory 10(1):98-107

Lopes AM, Machado JAT (2016) Integer and fractional-order entropy analysis of earthquake data series. Nonlinear Dynam 84(1):79-90

Macdonald JR (2015) “CNLS (complex nonlinear least squares) immittance, inversion, and simulation fitting programs for wINDOWS and MS-DOS: LEVM/LEVMW Manual". Available Online:
http://jrossmacdonald.com/jr-m/wpcontent/uploads/LEVMMAN UAL.pdf

Macdonald JR (1987) Impedance spectroscopy emphasizing solid materials and systems. Wiley, New York

Machado JAT, Jesus IS (2004) A suggestion from the past? J Fract Calc Appl Anal 7(4):403-407

Magin RL (2004) Fractional calculus in bioengineering, part 1. Crit Rev Biomed Eng 32(1):1-104

Magin R, Ortigueira MD, Podlubny I, Trujillo J (2011) On the fractional signals and systems. Signal Process 91(3):350-371

Melicio R, Catalao J, Mendes V (2010) "Fractional-order control and simulation of wind turbines with full-power converters". In: $15^{\text {th }}$ IEEE Mediterranean Electrotechnical Conf., Valletta, Malta, pp 320-325

Mishra S, Purwar S (2014) "To design optimally tuned FOPID controller for twin rotor мIMO system". In: Students Conf. Engineering and Systems

Mondal D, Biswas K (2011) Performance study of fractional order integrator using single-component fractional order element. IET Circuits Devices Syst 5(4):334-342

Mondal D, Biswas K (2013) Packaging of single component fractional order element. IEEE Trans Device Mater Rel 13(1):73-80

Mondal D (2012) "Fabrication and performance studies of PMMA coated fractional order elements". Master's thesis, Indian Inst. of Technol. Kharagpur, India, Dept. Electrical Eng

Monje C, Chen Y, Vinagre B, Xue C, Feliu V (2010) Fractional-order Systems and Controls. Springer, Berlin

Oldham KB, Spanier J (1974) The fractional calculus theory and applications of differentiation and integration to arbitrary order. Academic Press, New York

Ortigueira M (2011) Fractional calculus for scientists and engineers. Springer, Berlin

Oustaloup A, Levron F, Mathieu B, Nanot F (2000) Frequency band complex non integer differentiator: characterization and synthesis. IEEE Trans Circuits Syst I 47(1):25-40

Ozdemir N, Iskender BB (2010) Fractional order control of fractional diffusion systems subject to input hysteresis. J Comput Nonlinear Dyn 5(2):021002

Pinto CMA, Carvalho RM (2014) New findings on the dynamics of HIV and TB coinfection models. Appl Math Comput 242:36-46

Podlubny I (1999) Fractional-order systems and $\mathrm{pi}^{\lambda} \mathrm{d}^{\mu}$-controllers. IEEE Trans Autom Control 44(1):208-214

Radwan AG, Elwakil AS, Soliman AM (2008) Fractional-order sinusoidal oscillators: design procedure and practical examples. IEEE Trans Circuit Syst I 55(7):2051-2063

Roy SCD (1967) "On the realization of a constant-argument immitance or fractional operator". IEEE Trans Circuit Theory CT-14:264-274

Saha S, Das S, Ghosh R, Goswami B, Balasubramanian R, Chandra A, Das S, Gupta A (2010) Design of a fractional order phase shaper for iso-damped control of a phwr under step-back condition. IEEE Trans Nuclear Sci 27(3):1602-1612

Sapoval B, Gutfraind R, Meakin P, Keddam M, Takeounti H (1993) Equivalent-circuit, scaling, random-walk simulation, and an experimental study of self-similar fractal electrodes and interfaces. Phys Rev E 48(5):3333-44

Sierociuk D, Podlubny I, Petras I (2013) Experimental evidence of variable-order behavior of ladders and nested ladders. IEEE Trans Control Syst Technol 21(2):459-466

Sierociuk D, Skovranek T, Macias M, Podlubny I, Petras I, Dzielinski A, Ziubinski P (2015) Diffusion process modeling by using fractional-order models. Appl Math Comput 257:2-11

Soltan A, Radwan AG, Soliman AM (2015) Fractional order sallen key and khn filters: stability and poles allocation. Circuits Syst Signal Process 34(5):1461-80 
Tripathy M, Biswas K, Sen S (2013) A design example of a fractional-order kerwin- huelsman- newcomb biquad filter with two fractional capacitors of different order. Circuits Syst Signal Process 32(4):1523-1536

Tripathy MC, Mondal D, Biswas K, Sen S (2015) Experimental studies on realization of fractional inductors and fractional-order bandpass filters. Int J Circ Theor Appl 43(9):1183-1196

Tripathy MC, Mondal D, Biswas K, Sen S (2015) Design and performance study of phase-locked loop ( plls) using fractionalorder loop filter. Int J Circ Theor Appl 43(6):776-792
Tseng CC (2007) Design of FIR and IIR fractional order simpson digital integrators. Signal Process 87(5):1045-1057

Westerlund S, Ekstam L (1994) Capacitor theory. IEEE Trans Dielectr Electr Insul 1(5):826-839

Žecov M, Terpák J (2015) Heat conduction modeling by using fractional-order derivatives. Appl Math Comput 257:365-373 